\title{
Die Dysarthrie des Morbus Parkinson: Klinische Präsentation, pathophysiologische und diagnostische Aspekte
}

\section{Dysarthria in Parkinson's Disease: Clinical Presentation, Pathophysiological and Diagnostic Aspects}

\author{
Autor \\ S. Skodda \\ Institut \\ Neurologische Universitätsklinik Knappschaftskrankenhaus
}

Schlüsselwörter

- Morbus Parkinson

hypokinetische Dysarthrie

- Dopaminmangel

- Stimm-/Sprechtherapie

Key words

- parkinson's disease

- hypokinetic dysarthria

- dopaminergic deficit

- speech therapy

\section{Bibliografie}

DOI http://dx.doi.org/

10.1055/s-0041-102793

Sprache · Stimme - Gehör

2015; 39: 182-186

(c) Georg Thieme Verlag KG

Stuttgart · New York

ISSN 0342-0477

Korrespondenzadresse

PD Dr. med. Sabine Skodda

Neurologische Klinik der

Ruhr-Universität Bochum

Knappschaftskrankenhaus

In der Schornau 23-25

44892 Bochum

sabine.skodda@kk-bochum.de

\section{Zusammenfassung}

$\nabla$

Bei der hypokinetischen Dysarthrie des Morbus Parkinson handelt es sich um eine multidimensionale Störung, die bei der Mehrzahl der Patienten zu einer relevanten Kommunikationsstörung und damit Beeinträchtigung der Lebensqualität führen kann. Neben dem pathognomonischen Befund einer leisen, monotonen Sprechweise kommt es in unterschiedlichem Ausmaß auch zu Beeinträchtigungen von Sprechatmung, Stimmbildung (Phonation), Aussprache (Artikulation), Sprechgeschwindigkeit und -rhythmus. Muster und Ausmaß der Dysarthrie können individuelle Unterschiede aufweisen und zeigen im Krankheitsverlauf eine Progressionstendenz. Die Pathophysiologie ist bislang nicht vollständig bekannt, ist aber nicht ausschließlich durch einen Dopaminmangel zu erklären. Therapie der Wahl ist eine Stimm-/Sprechtherapie. Die Diagnostik beruht im klinischen Alltag zumeist auf einer perzeptuellen Analyse, die je nach individueller Fragestellung um akustische Analyse, physiologische Messungen und/oder funktionelle Bildgebung ergänzt werden kann.

\section{Abstract \\ $\nabla$}

Hypokinetic dysarthria of Parkinson's disease is a multidimensional impairment associated with considerable communication deficits with great impact on quality of life in the majority of patients. The typical pattern of Parkinsonian dysarthria includes a reduction in loudness and intonation variability ("monopitch and monoloudness") often combined with impaired speech respiration, phonation, articulation and temporal aspects of speech such as speech rate and rhythm. Pattern and degree of dysarthria can differ to some extent from individual to individual and show progressive deterioration over the course of disease progression. The underlying pathophysiology is still not fully understood; however, it cannot be explained by dopaminergic deficits alone. Speech/language therapy is the treatment of choice for Parkinsonian dysarthria. The diagnostic approach is based on perceptual analysis of speech in the clinical setting, but can be complemented by acoustic analyses, physiologic measures and functional neuro-imaging.

\section{Lernziel}

Durch die Lektüre des Artikels soll der Leser die klinische Präsentation der Parkinson-typischen Dysarthrie und ihre Entwicklung im Krankheitsverlauf sowie die zugrundeliegende Pathophysiologie kennenlernen.

\section{Einleitung}

Beim Morbus Parkinson (MP) handelt es sich um eine sporadische neurodegenerative Erkrankung, an der in Deutschland über 350000 Menschen leiden. Klinisch ist der Morbus Parkinson charakterisiert durch motorische Auffälligkeiten, näm- lich durch die Kombination einer Akinese mit Rigor, Tremor und/oder posturaler Instabilität. Diese motorischen Kernsymptome, die im Wesentlichen durch einen Verlust domaninproduzierender Zellen in der Substantia nigra des Mittelhirns verursacht sind, beginnen subtil, nehmen aber im Verlauf von wenigen Jahren kontinuierlich zu. Sie können schließlich im Verlauf sämtliche Bewegungsabläufe einbeziehen und beeinträchtigen ([22], ๑ Tab. 1). Das medikamentöse Therapiekonzept des MP beruht darauf, den Dopaminmangel im Bereich der Basalganglien mittels dopaminagonistisch wirksamer Pharmaka auszugleichen. Allerdings ist anhand neuropathologischer Untersuchungen 
inzwischen nachgewiesen, dass die neurodegenerativen Veränderungen weit über die Schädigung der Substantia nigra hinausgehen und nicht sämtliche Krankheitssymptome durch einen Dopaminmangel, sondern durch die Dysfunktion weiterer, z.T. noch nicht präzise definierter neuronaler Netzwerke und Neurotransmitter verursacht sind $([1,22,23] \odot$ Tab. 1).

Als ein weiteres charakteristisches Symptom des Morbus Parkinson leiden Betroffene im Krankheitsverlauf in bis zu $90 \%$ an Stimm- und Sprechstörungen, die als hypokinetische Dysarthrie (oder Dysarthrophonie, was den Aspekt der Stimm-Störung besser widerspiegelt) bezeichnet werden [2-4].

\section{Die hypokinetische Dysarthrie des Morbus Parkinson \\ $\nabla$}

Klinisches Bild

Erste Beschreibung

Systematische Untersuchungen der Parkinson-typischen Dysarthrie wurden erstmalig 1969 durch die Arbeitsgruppe um Darley durchgeführt, wobei mittels qualitativer perzeptueller Analyse ein typisches Muster von Sprechauffälligkeiten definiert wurde, das als „hypokinetische“ Dysarthrie zusammengefasst werden kann. Charakteristische Merkmale sind demnach eine Reduktion der Sprechlautstärke (Hypophonie), eine Einschränkung der Tonhöhen- und Lautstärke-Modulation („monopitch and monoloudness"), eine Verringerung der Akzentuierung („reduced stress“), gestörte Präzision in der Artikulation von Konsonanten, raue und behauchte Stimmqualität sowie variables Sprechtempo, z.T. mit unangemessenen Sprechpausen im Wechsel mit kurzen „Sprechschüben“ („,rush“) [4,5,24].

\section{Weitere Untersuchungen}

Seitdem sind zahlreiche weitere Untersuchungen zu unterschiedlichen Aspekten der Parkinson-Dysarthrie publiziert. Insbesondere die pathognomonischen klinischen Auffälligkeiten der Hypophonie und Monotonie ließen sich übereinstimmend als eingeschränkte Modulationsfähigkeit von Tonhöhenverlauf und Lautstärke im Verlauf des Sprechens objektivieren [5,6, 25-27]. Darüber hinaus wurde bei der Mehrzahl der untersuch- ten Patienten eine Phonationsstörung identifiziert, einhergehend mit einer Verringerung der durchschnittlichen Sprechlautstärke, einer erhöhten durchschnittlichen Tonhöhe sowie einem höheren Anteil von Mikroperturbationen als Maß einer laryngealen Instabilität, was sich klinisch durch eine raue oder heisere Stimmqualität manifestieren kann $[6,7,28,29]$.

Diese Dysphonie wurde pathophysiologisch zurückgeführt auf eine dysfunktionale Aktion agonistischer und antagonistischer Muskelgruppen mit daraus resultierender verminderter Bewegungsamplitude.

\section{Störungen der Sprechatmung}

Die Beeinträchtigungen der Stimmbildung können durch zusätzliche Störungen der Sprechatmung beim MP verstärkt werden, was z.B. in einer verringerten Anzahl der produzierten Silben pro Atemzug oder einer Reduktion der maximalen Vokalhaltedauer resultieren kann $[8,30]$. Als ursächlich für die insuffiziente Sprechatmung wird meist ein erhöhter Widerstand des respiratorischen Systems angesehen, ausgelöst durch Rigor und Hypokinese der Atemmuskulatur. Diese Mechanismen werden meist auch als Erklärung für die laryngealen Funktionsstörungen angesehen, wobei aber auch zusätzlich Indizien für pathologische zentrale Atemmuster beim MP gefunden wurden [31-33].

\section{Beeinträchtigung der Artikulation}

Ein weiteres klinisches Merkmal der hypokinetischen Dysarthrie ist die Beeinträchtigung der Artikulation, einhergehend mit einer verwaschen wirkenden, unpräzisen Aussprache. Auch dieser Aspekt der Parkinson-Dysarthrie kann als rigor- und/oder hypokinese-bedingte Reduktion des Bewegungsumfanges der Artikulatoren (d.h. Muskulatur von Zunge, Lippen und Kiefer) gedeutet werden, mit konsekutiven Einschränkungen von Umfang und Geschwindigkeit der Bewegungsabläufe $[35,36]$. So konnte bspw. gezeigt werden, dass Parkinson-Patienten in einer experimentellen Sprechsituation eine vorgegebene Sprechrate auf Kosten der Bewegungsamplitude aufrecht zu halten versuchten, im Sinne einer - insuffizienten - Kompensation der krankheitsbedingten Minderbeweglichkeit der Artikulatoren
Tab. 1 Symptome des Morbus Parkinson.

\section{motorische Symptome (asymmetri- \\ sche Präsentation typisch) \\ Hypokinese/Akinese (Störung von Initiierung, Durchführung und Beibe- haltung von Bewegungen, vorschnelle Ermüdung) Bradykinese (Verlangsa- mung von Bewegungen) \\ Rigor (erhöhter Muskeltonus, sog. „Zahnradphänomen“) \\ Tremor (Zittern, v. a. in Ruhe auftre- tend, Frequenz 4-6 Hz)}

Haltungsstörung (in späteren Stadien)

Dysphagie (in späteren Stadien)

Dysarthrie und Dysphonie

\section{Beispiele}

Störung der Fingerfeinmotorik, Mikrografie, vermindertes Mitschwingen der Arme beim Gehen, kleinschrittiges, verlangsamtes Gangbild ...

Steifigkeitsgefühl der Extremitäten, z.T. ziehende, krampfartige Schmerzen

meist Hände/Arme; Beine, Kopf und Kinn können betroffen sein

Gleichgewichtsverlust beim Gehen, vornüber und/oder zur Seite geneigte Körperhaltung Verschlucken, Gewichtsverlust, Aspiration nicht-motori-

sche Symptome

neuropsychiatrische Symptome

Schlafstörungen

autonome Funktionsstörungen

Sonstiges

\section{Beispiele}

Ängstlichkeit, Depression, verminderte psychophysische Leistungsfähigkeit, kognitive Defizite bis hin zum Vollbild einer Demenz in späteren Stadien, Apathie ...

REM-Schlaf-Verhaltensstörung, periodische Beinbewegungen im Schlaf, Durchschlafstörungen aufgrund nächtlicher Akinese ... Miktionsstörungen, erektile Dysfunktion, Kreislaufdysregulation, Obstipation, Hyperhidrose ...

Hyposmie, „Salbengesicht“ ... 
[9]. Dieses Phänomen des „articulatory undershooting“ beeinflusst nicht nur die Artikulation der Konsonanten, sondern führt auch zu einer verminderten Präzision der Formantenbildung und damit der Vokalartikulation $[37,38]$.

\section{Unregelmäßigkeiten des Sprechtempos}

Als weitere Auffälligkeit sind Unregelmäßigkeiten des Sprechtempos zu nennen, die interindividuell allerdings sehr unterschiedlich ausgeprägt sein können. In auf Silbenrepetition beruhenden Sprechtests wurden sowohl pathologisch beschleunigte als auch verlangsamte Repetitionsraten beschrieben, wobei insbesondere die Akzelerationstendenz bei einer Subgruppe von Patienten als Verlust der willkürlichen Kontrolle der Sprechgeschwindigkeit interpretiert wurde [10,39-41]. Ein ähnlich heterogenes Bild zeigte sich auch in Untersuchungen, die auf komplexeren Sprechtests basierten: Neben verlangsamten und erhöhten Sprechraten wurden auch normale Sprechgeschwindigkeiten erhoben, zum Teil einhergehend mit Veränderungen von Anzahl und Dauer der Sprechpausen als Hinweis für eine Dysintegration der zeitlichen Organisation komplexer Sprechvorgänge [11]. Neben dem Vorkommen unphysiologisch langer Sprechpausen wurden bei anderen Patienten auch als Palilalie bezeichnete unwillkürliche Wort- oder Silbenwiederholungen beobachtet, die als Indiz für eine gestörte Sprechinhibition gelten $([5,42] \odot$ Tab. 2 finden Sie im Internet).

\section{Individuell unterschiedliche Ausprägung}

Allerdings gilt es zu berücksichtigen, dass Ausmaß und auch spezifisches Erscheinungsbild der Dysarthrie individuell unterschiedlich ausgeprägt sein können. Bspw. scheint es Subgruppen von Patienten zu geben, bei denen isolierte Störungen des Redeflusses initial ohne wesentliche Einschränkungen von Phonation und Artikulation auftreten [2].

Angesichts des chronisch fortschreitenden Charakters der Parkinson-Erkrankung kommt es außerdem im Verlauf der Erkrankung typischerweise zu einer Zunahme der Dysarthrie, was entsprechenden Einfluss auf die globale Kommunikationsfähigkeit und Lebensqualität der Patienten haben kann (z. B. [4, 5, 43,44]).

\section{Entwicklung der Dysarthrie im Krankheitsverlauf Frühstadium}

Anhand der Studien, die mittels Untersuchungen an ausreichend großen Patientenkollektiven dem chronisch-progredienten Verlauf des MP Rechnung tragen, wurde postuliert, dass die hypokinetische Dysarthrie im Frühstadium der Erkrankung mit Störungen der Phonation beginnt, gekennzeichnet durch Veränderungen von Stimmqualität und durchschnittlicher Tonhöhe sowie durch eine Reduktion von Tonhöhenvariabilität und mittlerer Sprechlautstärke $[2,11]$. Ebenso lässt sich eine verringerte Bewegungsamplitude der artikulatorischen Muskelgruppen bereits im frühen Krankheitsstadium beobachten. Insbesondere diese in der Frühphase auftretenden dysarthrischen Störungen, durch die es im Allgemeinen (noch) nicht zu einer Einschränkung der globalen Sprechverständlichkeit kommt, sprechen zum Teil auf die medikamentöse Therapie mit dopaminerg wirksamen Medikamenten an $[12,45,46]$.

\section{Spätstadium}

Nach längerem Krankheitsverlauf ist anhand der wenigen longitudinalen Untersuchungen eine quantitative Zunahme der Stö- rung von Phonation und Artikulation beschrieben [13]. Zusätzlich treten aber auch merkliche Beeinträchtigungen der Prosodie, und dabei insbesondere von Sprechgeschwindigkeit und -rhythmus auf, die vermutlich als Ausdruck einer Dysfunktion von zeitlicher Planung und Umsetzung sprechmotorischer Abläufe $\mathrm{zu}$ interpretieren sind [13]. Trotz zum Teil kontroverser Datenlage scheinen zumindest diese im Krankheitsverlauf in den Vordergrund tretenden Störungen nicht ausreichend auf dopaminerge Therapie anzusprechen $[3,13]$. Es ist sogar eine Verstärkung von Störungen des Redeflusses unter Medikation bei einzelnen Patienten beschrieben [14,47-49], was insgesamt dafür spricht, dass mit fortschreitender Neurodegeneration zunehmend komplexere und nicht allein durch einen Dopaminmangel begründete Mechanismen für die Ausprägung und das Ausmaß der Dysarthrie verantwortlich sind.

Dysarthrische Störungen in der Frühphase sprechen im Gegensatz zu den Störungen im weiteren Verlauf zum Teil auf die medikamentöse Therapie mit dopaminerg wirksamen Medikamenten an.

\section{Pathophysiologische Überlegungen \\ $\nabla$}

Der Dopaminmangel im Bereich der Basalganglien und die daraus resultierende Störung ihrer Interaktion mit kortikalen Zentren hat insbesondere für die offensichtlichen motorischen Störungen eine herausragende Bedeutung. Allerdings ist die Pathophysiologie des MP insbesondere in weiter fortgeschrittenen Erkrankungsstadien sehr viel komplexer und bezieht auch andere Hirnareale und Dysbalancen anderer Neurotransmitter mit ein, was erklärt, warum neben den motorischen Kernsymptomen noch zahlreiche andere Störungen auftreten können [1,50,51]. Zusätzlich muss berücksichtigt werden, dass Krankheitserscheinungen, die zu einem bestimmten Zeitpunkt bei einem Patienten beobachtet werden, durch den Einfluss der Medikation und/ oder überlagernde (und zum Teil ebenfalls wieder dysfunktionale) Kompensationsmechanismen verändert oder maskiert werden können. Dies gilt besonders auch für das pathophysiologische Verständnis der Parkinson-Dysarthrie, die als multidimensionale progrediente Störung in unterschiedlichem Ausmaß statische wie dynamische Sprechparameter einbezieht. Phänomenologisch können unterschiedliche Teilaspekte definiert werden, die Hypothesen über die zugrundeliegende Pathophysiologie erlauben.

\section{Motorische Ebene}

Ein wesentlicher Aspekt der hypokinetischen Dysarthrie betrifft die motorische Ebene mit einer Reduktion des Bewegungsumfanges (Hypokinese), einer pathologischen Skalierung und einer insuffizienten Beibehaltung repetitiver Sprechbewegungen, einhergehend mit einer Tendenz, die Amplitude von Beginn an fehlerhaft zu definieren bzw. im Verlauf der Ausführung weiter zu reduzieren (z.B. $[9,15,52,53])$. Dies ist die Grundlage für die beobachtete Reduktion von Sprechlautstärke und Tonhöhenvariabilität mit einer weiteren Abnahme im Verlauf des Sprechens. Eine zusätzliche fehlerhafte Planung und Umsetzung der zeitlichen Abfolge von Sprechbewegungen manifestiert sich bspw. in der unwillkürlichen Reduktion oder auch Akzeleration bei Sprechen (z. B. $[3,4,9,10])$. In diesem Zusammenhang sind auch Störungen des internen „cueing“ von Bedeutung, die dadurch demaskiert werden, dass dysarthrische Parkinson-Patienten ihre 
Sprechverständlichkeit, -geschwindigkeit und -lautstärke in Abhängigkeit von der Sprechaufgabe und externen Aufforderungen oder Taktgebern zumindest kurzzeitig relevant verbessern können (z.B. $[3,16,54,55])$. Diese Phänomene sind vermutlich insbesondere auf die Parkinson-typische Dysfunktion der Basalganglien zurückzuführen, die physiologischerseits an der Planung, Initiierung, Stabilisierung und Aufrechterhaltung quasi-automatisierter motorischer Abläufe maßgeblich beteiligt sind [56].

\section{Eingeschränkte sensorische Kontrolle}

Zusätzlich gibt es zahlreiche Indizien dafür, dass auch eine eingeschränkte sensorische Kontrolle - in Form von eingeschränkter Kinästhesie, Propriozeption und Feedback-Kontrolle - für die Parkinson-Dysarthrie von Bedeutung ist [57,58]. Dies führt am Sprechapparat bspw. zu einer fehlerhaften Einschätzung von Kraft und Bewegungsamplitude und wird durch Störungen der Selbstwahrnehmung von Lautstärke und Geschwindigkeit weiter verstärkt $[17,59]$. Eine daraus resultierende Miniaturisierung der Sprechbewegungen trägt wesentlich zum Aspekt der Hypokinese bei und betrifft insbesondere automatisiert ablaufende Bewegungsmuster, für deren Aufrechterhaltung üblicherweise keine gerichtete Aufmerksamkeit oder Überwachung erforderlich ist (z. B. [18]). Es wird angenommen, dass die Ursache dafür in einer inadäquaten „Zusammenarbeit“ zwischen dysfunktionalen Basalganglien und Thalamus mit für die Sprechvorgänge bedeutsamen kortikalen Arealen liegen könnte [3]. Dieses Konzept findet indirekte Bestätigung dadurch, dass gestörte und unterskalierte Sprechbewegungsabläufe durch eine Erhöhung der „vocal vigilance“ mittels Training wieder der bewussten Kontrolle und Überwachung unterworfen, dadurch deautomatisiert und letztlich verbessert werden können. Dieses Konzept lässt sich therapeutisch nutzen, indem in einer speziellen Sprechtherapie (Lee-Silverman-Voice-Treatment/LSVT ${ }^{\circledR}$ ) bei dysarthrischen Parkinson-Patienten die Aufmerksamkeit insbesondere auf den Aspekt der Sprechlautstärke gerichtet wird, mit dem Ziel, zunächst eine bewusste Kontrolle und schließlich eine „Neuskalierung“ des Sprechvorgangs zu erzielen. Dies kann zumindest bei einigen Patienten zu einer erheblichen Verbesserung der hypokinetischen Dysarthrie führen (z. B. [19,60,61]).

\section{Kognitive Einschränkungen}

Allerdings kommt es im Rahmen der fortschreitenden ParkinsonErkrankung bei vielen Betroffenen auch zu kognitiven Einschränkungen, die die Fähigkeit der willentlichen Fokussierung auf eine Kontrolle und Anpassung der eigenen (Sprech-)Bewegungsabläufe zusätzlich beeinträchtigen können. Insbesondere Störungen von Konzentration, gerichteter und geteilter Aufmerksamkeit sowie Umstellungsfähigkeit und die nachlassende Fähigkeit zum „multi-tasking“, die zusammengefasst als Dysexekutivsyndrom bezeichnet werden, können die Wahrnehmung und Verbesserungsfähigkeit des eigenen Sprechvorgangs zusätzlich negativ beeinflussen $[20,62]$.

Diese neuropsychologischen Defizite können sich insbesondere bei älteren Patienten nach langjährigem Krankheitsverlauf zum Vollbild einer Demenz entwickeln, so dass neben der Dysarthrie noch zusätzliche Sprachstörungen (durch die Beeinträchtigung von sprachlicher Konzeptbildung, Wortfindung, Sprachverständnis usw.) hinzutreten und die Kommunikationsfähigkeit dramatisch verschlechtern können [63].

\section{Diagnostische Aspekte}

\section{$\nabla$}

\section{Perzeptuelle Analyse}

Für die Diagnostik der Parkinson-Dysarthrie stehen unterschiedliche Verfahren zur Verfügung, die je nach zugrundeliegender Fragestellung allein oder in Kombination zum Einsatz kommen können. Die perzeptuelle Analyse orientiert sich an dem vom Patienten produzierten Sprechsignal, was dann durch den Untersucher erfasst und qualitativ beschrieben wird. Neben der globalen Sprechverständlichkeit können die einzelnen Subsysteme des Sprechens - wie Atmung, Phonation, Artikulation, Prosodie usw. - dabei genauer analysiert werden, wobei naturgemäß die Vergleichbarkeit der Ergebnisse intra- und inter-individuellen Schwankungen unterliegen kann. Im klinischen Alltag ist die perzeptuelle Analyse durch einen professionellen Untersucher sicher die am meisten verbreitete diagnostische Maßnahme und dient zur Erfassung des Ausmaßes einer Dysarthrie und zur Überprüfung von Therapieerfordernis und -kontrolle. In Kombination mit Selbst- und Fremdbeurteilungsinstrumenten, meist in Form standardisierter Fragebögen, kann außerdem das für den Patienten bedeutsame Ausmaß der aus der Dysarthrie resultierenden globalen Kommunikationsstörung und Behinderung erfasst werden $[64,65]$.

\section{Akustische Analyse}

Die Methodik der akustischen Analyse, die ebenfalls vom akustischen Sprechsignal ausgeht, ersetzt die Expertise des „hörgeschulten“ Untersuchers durch die computerbasierte, meist automatisierte Auswertung zahlreicher Eigenschaften des akustischen Signals und liefert damit objektive und quantifizierbare Messwerte. Die Stärke der Methode liegt neben ihrer Untersucherunabhängigkeit vermutlich darin, dass auch subtile Veränderungen des Sprechsignals detektiert werden können, die der perzeptuellen Analyse entgehen. Da mittels akustischer Analyse diskrete und oftmals subklinische Stimm- und Sprechveränderungen bereits in frühen Erkrankungsstadien detektiert werden können, liegt hier ein Potenzial für die Früh- und/oder Differenzialdiagnostik von Parkinson-Syndromen (z.B. $[10,66])$. Ebenso könnte sich die computerunterstützte Erfassung bestimmter Sprechparameter als Verlaufsparameter insbesondere für nicht-dopaminerge Krankheitsprogression etablieren [13]. Allerdings hat sich bislang kein akustischer Einzelparameter etabliert, der die Resultate der „globalen Sprechverständlichkeit“ oder den Aspekt der funktionellen Beeinträchtigung ausreichend abbildet, so dass perzeptuelle und akustische Analyse am ehesten als komplementär anzusehen sind.

\section{Physiologische Methoden}

Physiologische Methoden zielen hingegen darauf ab, die dem Sprechsignal zugrundeliegenden „Effektororgane“, also die Atem-, Mund- und Schlundmuskulatur sowie die Stimmlippen, in Ruhe und Bewegung zu untersuchen, um Funktionsstörungen zu detektieren und quantitativ zu erfassen. Hierzu zählen bspw. videostroboskopische oder fiberendoskopische Untersuchungen der Stimmlippenbewegung bei der Phonation oder kinematische oder elektrophysiologische Messungen der Lippen-, Zungen- und Kieferbewegung bei der Artikulation. Da diese Diagnostik meist den z.T. invasiven Einsatz unterschiedlich aufwändiger Apparaturen erfordert, bildet sie im Einzelfall allerdings oftmals nicht den „natürlichen“ Sprechablauf ab. 


\section{Funktionelle Bildgebung}

Insbesondere für das Verständnis der Pathophysiologie von neurogenen Dysarthrien kommt in jüngerer Zeit die funktionelle Bildgebung, z.B. in Form des funktionellen MRT, zum Einsatz, mit dem man zerebrale Areale und/oder Funktionssysteme darstellen kann, die bei der gerade während der Untersuchung durchgeführten Sprechaufgabe aktiviert sind. Diese Art der Diagnostik ist aktuell aber wissenschaftlichen Fragestellungen vorbehalten.

\section{Zugrundeliegende Sprechaufgabe}

Neben der Wahl der diagnostischen Methode ist auch die Art der zugrundeliegenden Sprechaufgabe für die Einschätzung der vorliegenden Funktionsstörung von großer Bedeutung. So ist es insbesondere für die Parkinson-Dysarthrie bekannt, dass Art und Kontext des Sprechens einen großen Einfluss auf die globale Sprechverständlichkeit haben können (z.B. $[16,18])$. Ebenso muss beachtet werden, dass die Ergebnisse von Untersuchungen, die bspw. auf der Durchführung basaler "nicht-sprachlicher“ Silbenrepetitionen basieren, nicht automatisch Rückschlüsse auf die Funktion komplexer sprachlicher Prozesse erlauben.

Diese hier kurz dargestellten diagnostischen und methodischen Aspekte machen deutlich, dass die Auswahl der im Einzelfall sinnvollen Diagnostik entscheidend von der zugrundeliegenden Fragestellung abhängt und dies auch bei der Interpretation und Bewertung wissenschaftlicher Daten berücksichtigt werden muss.

\section{Fazit}

- Die Mehrzahl der Patienten mit Morbus Parkinson entwickeln im Verlauf der Erkrankung eine Dysarthrie.

- Das charakteristische Muster der hypokinetischen Dysarthrie/Dysarthrophonie ist charakterisiert durch

- eine Verschlechterung der Stimmqualität (raue, behauchte, z.T. zittrige Stimme),

- eine Reduktion von Lautstärke (Hypophonie) und verringerte Modulation von Satzmelodie und Sprechintensität,

- eine Abnahme der Artikulationsschärfe von Konsonanten und Vokalen und

- Veränderungen von Sprechgeschwindigkeit und -rhythmus.

- Die zugrundeliegende Pathophysiologie ist komplex und erst unzureichend geklärt; nicht alle Teilaspekte der Dysarthrie können durch ein dopaminerges Defizit erklärt werden.

- Die Therapie der hypokinetischen Dysarthrie ist oftmals unbefriedigend:

- Dopaminerge Medikation hat insbesondere im späteren Krankheitsverlauf keine befriedigende Wirkung.

- Stimm-/Sprachtherapie gilt als die Therapie der Wahl, nach aktueller Datenlage scheint LSVT $^{\circledR}$ wirksam zu sein.

- Die pragmatische Therapie der Dysarthrie muss sich nach dem Ausmaß der Beeinträchtigung und der individuellen Krankheitssituation des einzelnen Patienten richten.

\section{Ergänzendes Material}

\section{$\nabla$}

Die Tab. 2 finden Sie im Internet unter http://dx.doi.org/ 10.1055/s-0041-102793.
Zur Person

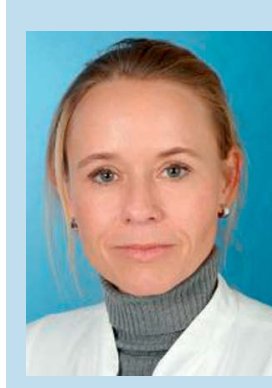

PD Dr. med. Sabine Skodda ist leitende Oberärztin in der Neurologischen Klinik am Universitätsklinikum Knappschaftskrankenhaus Bochum. Ihr klinischer Arbeitsschwerpunkt betrifft extrapyramidale Bewegungsstörungen, insbesondere die Diagnostik und Therapie des Morbus Parkinson und verwandter Erkrankungen. Wissenschaftlich befasst sie sich mit der Analyse von Stimm- und Sprechstörungen bei Morbus Parkinson.

Interessenkonflikt: Die Autorin gibt an, dass kein Interessenkonflikt besteht.

\section{Literatur}

1 Farlow J, Pankratz ND, Wojcieszek J et al. Parkinson Disease Overview. In: Pagon RA, Adam MP, Ardinger HH et al. Eds. Seattle (WA); University of Washington: Seattle; 1993-2015 [Letztes Update 27.2.2014]

2 Ho A, Iansek R, Marigliani C et al. Speech impairment in a large sample of people with Parkinson's disease. Behav Neurol 1998; 11: 131-137

3 Sapir S. Multiple Factors Are Involved in the Dysarthria Associated With Parkinson's Disease: A Review With Implications for Clinical Practice and Research. JSLHR 2014; 57: 1330-1343

4 Duffy JR. Hypokinetic dysarthria. In: Duffy JR. (Ed.). Motor Speech Disorders, substrates, differentials diagnosis and management. St. Louis: Elsevier Mosby; 2005: 187-215

5 Darley FL, Aronson AE, Brown JR. Clusters of deviant speech dimensions in the dysarthrias. J Speech Hear Res 1969; 12: 462-496

6 Goberman AM, Coelho C. Acoustic analysis of parkinsonian speech I: speech characteristics and L-Dopa therapy. NeuroRehabilitation 2002; 17: 237-246

7 Holmes RJ, Oates J, Phyland D et al. Voice characteristics in the progression of Parkinson's disease. Int J Lang Commun Disord 2000; 35: 417-418

8 Huber JE, Spruill J. Respiratory function and variability in individuals with Parkinson's disease: pre- and post-Lee Silverman Voice Treatment. J Med Speech Lang Pathol 2003; 11: 185-192

9 Ackermann H, Konczak J, Hertrich I. The temporal control of repetitive articulatory movements in Parkinson's disease. Brain Lang 1997; 57: 312-319

10 Skodda S. Aspects of speech rate and regularity in Parkinson's disease. J Neurol Sci 2011; 310: 231-236

11 Rusz J, Cmejla R, Růžičcková $H$ et al. Evaluation of speech impairment in early stages of Parkinson's disease: a prospective study with the role of pharmacotherapy. J Neural Transm 2013; 120: 319-329

12 Ho AK, Bradshaw JL, Iansek R. For better or worse: The effect of levodopa on speech in Parkinson's disease. Mov Disord 2008; 15 23: 574-580

13 Skodda S, Grönheit W, Mancinelli $N$ et al. Progression of voice and speech impairment in the course of Parkinson's disease: a longitudinal study. Parkinsons Dis 2013; 2013: 389195

14 Goberman AM, Blomgren M. Parkinsonian speech disfluencies: effects of L-dopa-related fluctuations. J Fluency Disord 2003; 28: 55-70

15 Ho AK, Iansek R, Bradshaw JL. Motor instability in Parkinsonian speech intensity. Neuropsychiatry Neuropsychol Behav Neurol 2001; 14: 109-116

16 Goberman AM, Elmer WE. Acoustic analysis of clear versus conversional speech in individuals with Parkinson's disease. J Commun Disord 2005; 38: 215-230

17 Ho AK, Bradshaw JL, Iansek $R$ et al. Speech volume regulation in Parkinson's disease: effects of implicit cues and explicit instructions. Neuropsychologia 1999; 37: 1453-1460

18 Kempler D, Van Lancker $D$. Effect of speech task on intelligibility in dysarthria: a case study of Parkinson's disease. Brain Lang 2002; 80: 449-464

19 Fox CM, Ramig LO, Ciucci MR et al. The science and practice of LSVT/ LOUD: neural plasticity-principled approach to treating individuals with Parkinson disease and other neurological disorders. Semin Speech Lang 2006; 27: 283-299

20 McNamara $P$, Obler $L K, A u R$ et al. Speech monitoring skills in Alzheimer's disease, Parkinson's disease, and normal aging. Brain Lang 1992; 42: 38-51 
21 Poewe W. The natural history of Parkinson's disease. J Neurol 2006; 253 Suppl 7: VII2-6

22 Braak H, Del Tredici K, Rüb U et al. Staging of brain pathology related to sporadic Parkinson's disease. Neurobiol Aging 2003; 24: 197-211

23 Jellinger KA. Synuclein deposition and non-motor symptoms in Parkinson disease. J Neurol Sci 2011; 310: 107-111

24 Darley FL, Aronson AE, Brown JR. Differential diagnostic patterns of dysarthria. J Speech Hear Res 1969; 12: 246-269

25 Metter J, Hanson W. Clinical and acoustical variability in hypokinetic dysarthria. J Commun Disord 1986; 19: 347-366

26 Skodda S, Visser W, Schlegel U. Gender-related patterns of dysprosody in Parkinson's disease and correlation between speech variables and motor symptoms. J Voice 2011; 25: 76-82

27 Logemann JA, Fisher HB, Boshes B, et al. Frequency and co-occurrence of vocal tract dysfunction in the speech of a large sample of parkinsonian patients. J Speech Hearing Disord 1978; 43: 47-57

28 Hertrich I, Ackermann H. Gender-specific vocal dysfunction in Parkisnon's disease: Electroglottographic and acoustical analyses. An Oto Rhino Laryngol 1995; 104: 197-202

29 Tanaka Y, Nishio M, Niimi S. Vocal acoustic characteristics of patients with Parkinson's disease. Folia Phoniatr Logop 2011; 63: 223-230

30 Hovestadt A, Bogaard JM, Meerwaldt J et al. Pulmonary function in Parkinson's disease. J Neurol Neurosurg Psychiatry 1989; 52: 329-333

31 Baker KK, Ramig LO, Luschei ES, et al. Thyroarytenoid muscle activity associated with hypophonia in Parkinson's disease and aging. Neurology 1998; 51: 1592-1598

32 Hanson DG, Gerrat BR, Ward PH. Cinegraphic observations of laryngeal function in Parkinson's disease. Laryngoscope 1984; 94: 348-353

33 Perez KS, Ramig LO, Smith ME, et al. The parkinson larynx: tremor and videoscopic findings. J Voice 1996; 10: 354-361

34 McClean MD, Beukelman DR, Yorkston KM. Speech-muscle visuomotor tracking in dysarthric and nonimpaired speakers. J Speech Hear Res 1987; 30: 276-282

35 Forrest K, Weismer G, Turner GS. Kinematic acoustic and perceptual analyses of connected speech produced by parkinsonian and normal geriatric adults. J Acoust Soc Am 1989; 85: 2608-2622

36 Hunker C, Abbs J, Barlow S. The relationship between parkinsonian rigidity and hypokinesia in the orofacial system: a quantitative analysis. Neurology 1982; 2: 749-754

37 Sapir S, Spielman JL, Ramig LO et al. Effects of intensive voice treatment (the Lee Silverman Voice Treatment [LSVT]) on vowel articulation in dysarthric individuals with idiopathic Parkinson disease: acoustic and perceptual findings. J Speech Lang Hear Res 2007; 50: 899-912

38 Skodda S, Visser W, Schlegel U. Vowel articulation in Parkinson's disease. J Voice 2011; 25: 467-472

39 Hirose H, Kiritani S, Sawashima M. Velocity of articulatory movements in normal and dysarthric subjects. Folia Phoniatr Logop 1982; 34: 210-215

40 Ludlow CL, Connor NP, Bassich CJ. Speech timing in Parkinson's and Huntington's disease. Brain Lang 1987; 32: 195-214

41 Flasskamp A, Kotz SA, Schlegel U, et al. Acceleration of syllable repetition in Parkinson's disease is more prominent in the left-side dominant patients. Parkinsonism Relat Disord 2012; 18: 343-347

42 Benke T, Butterworth B. Palilalia and repetitive speech: two case studies. Brain Lang 2001; 78: 62-81

43 Majdinasab F, Karkheiran S, Moradi N et al. Relation between Voice Handicap Index (VHI) and disease severity in Iranian patients with Parkinson's disease. Med J Islam Repub Iran 2012; 26:157-163

44 Miller N, Noble E, Jones D, et al. Life with communication changes in Parkinson's disease. Age Ageing 2006; 35: 235-239
45 Gallena S, Smith PJ, Zeffiro T, et al. Effects of levodopa on laryngeal muscle activity for voice onset and offset in Parkinson's disease. J Speech Lang Hear Res 2001; 44: 1284-1299

46 Goberman A, Coelho C, Robb M. Phonatory characteristics of parkinsonian speech before and after morning medication: the ON and OFF states. J Commun Disord 2002; 35: 217-239

47 Tykalová T, Rusz J, Čmejla R et al. Effect of dopaminergic medication on speech dysfluency in Parkinson's disease: a longitudinal study. J Neural Transm. 2015 Jan 13. [Epub ahead of print]

48 Louis ED, Winfield L, Fahn S, et al. Speech dysfluency exacerbated by levodopa in Parkinson's disease. Mov Disord 2001; 16: 562-565

49 Goberman AM, Blomgren M. Parkinsonian speech disfluencies: effects of L-dopa-related fluctuations. J Fluency Disord 2003; 28: 55-70

50 Obeso JA, Marin C, Rodriguez-Oroz C et al. The basal ganglia in Parkinson's disease: current concepts and unexplained observations. Ann Neurol 2008; 64 Suppl 2: S30-46

51 Gaig C, Tolosa E. When does Parkinson's disease begin? Mov Disord 2009; 24 (Suppl.2): S656-S664

52 Ackermann H, Ziegler W. Articulatory deficits in Parkinsonian dysarthria. J Neurol Neurosurg Psychiatry 1991; 54: 1093-1098

53 Caligiuri MP. The influence of speaking rate on articulatory hypokinesia in parkinsonian dysarthria. Brain Lang 1989; 36: 493-502

54 Sadagopan N, Huber JE. Effects of loudness cues on respiration in individuals with Parkinson's disease. Mov Disord 2007; 22: 651-659

55 Sapir S. Multiple factors are involved in the dysarthria associated with Parkinson's disease: a review with implications for clinical practice and research. J Speech Lang Hear Res 2014; 57: 1330-1343

56 Brown P, Marsden CD. What do the basal ganglia do? Lancet. 1998; 351: 1801-1804

57 Caligiuri M, Abbs JH. Response properties of the perioral reflex in Parkinson's disease. Exp Neurol 1987; 98: 563-572

58 Diamond SG, Schneider JS, Markham CH. Oral sensorimotor defects in people with Parkinson's disease. Adv Neurol 1987; 45: 335-338

59 Walshe M, Miller N, Leahy M, et al. Intelligibility of dysarthric speech: perceptions of speakers and listeners. Int J Lang Commun Disord 2008; 43: 633-648

60 Sapir S, Ramig L, Hoyt P et al. Phonatory-respiratory effort (LSVT ${ }^{\circledR}$ ) vs. respiratory effort treatment for hypokinetic dysarthria: comparing speech loudness and quality before and 12 months after treatment. Folia Phoniatr 2002; 54: 296-303

61 Spielman J, Ramig LO, Mahler L et al. Effects of an extended version of the lee silverman voice treatment on voice and speech in Parkinson's disease. Am J Speech Lang Pathol 2007; 16: 95-107

62 Rowe J, Stephan KE, Friston K et al. Attention to action in Parkinson's disease: impaired effective connectivity among frontal cortical regions. Brain 2002; 125(Pt 2): 276-289

63 Robbins TW, Cools R. Cognitive deficits in Parkinson's disease: a cognitive neuroscience perspective. Mov Disord 2014; 29: 597-607

64 Wertheimer J, Gottuso AY, Nuno M et al. The impact of STN deep brain stimulation on speech in individuals with Parkinson's disease: the patient's perspective. Parkinsonism Relat Disord 2014; 20: 1065-1070

65 Wight S, Miller N. Lee Silverman Voice Treatment for people with Parkinson's: audit of outcomes in a routine clinic. Int J Lang Commun Disord 2015; 50: 215-225

66 Rusz J, Cmejla R, Tykalova T et al. Imprecise vowel articulation as a potential early marker of Parkinson's disease: effect of speaking task. J Acoust Soc Am 2013; 134: 2171-81

67 Duffy JR. Hypokinetic dysarthria. In: Duffy JR, (Hrsg.). Motor Speech Disorders, substrates, differentials diagnosis and management. St. Louis: Elsevier Mosby; 2005: 187-215 